\section{Kidney \\ Blood Pressure Research}

\title{
Efficacy and Safety of Agents in IgA Nephropathy: An Update Network Meta- Analysis
}

\author{
Pingping Yang ${ }^{a, b}$ Qi Wang ${ }^{a}$ Chen Xie ${ }^{a}$ Gaosi Xu ${ }^{a}$ Qinghua Wu ${ }^{b}$ \\ aDepartment of Nephrology, the Second Affiliated Hospital of Nanchang University, Nanchang, \\ bDepartment of Cardiovascular Medicine, the Second Affiliated Hospital of Nanchang University, \\ Nanchang, China
}

\section{Key Words}

IgA nephropathy $\cdot$ Renin-angiotensin system $\bullet$ Steroid $\bullet$ Proteinuria

\begin{abstract}
Background/Aims: The present network meta-analysis of randomized controlled trials (RCTs) was to explore the efficacy and safety of different pharmacologic interventions in IgA nephropathy with proteinuria more than $0.75 \mathrm{~g} / \mathrm{d}$. Methods: We systematically searched the Cochrane Library, Embase, and PubMed database for studies compared the rate of clinical remission and/or serious adverse events in IgA nephropathy patients with proteinuria ( $>0.75$ $\mathrm{g} / \mathrm{d}$ ) up to August 1, 2018. We ranked the comparative effects of all drugs against placebo on the surface under the cumulative ranking area (SUCRA) probabilities. Results: There were 29 RCTs comprising 2517 participants included for the comparisons of 9 interventions. The rank of the most effective treatments for inducing clinical remission was renin-angiotensin system inhibitors (RASi) in combination with steroid, tonsillectomy combined with steroid pulse therapy, and azathioprine plus RASi with SUCRA of $82.9 \%, 80.5 \%$, and $67.6 \%$, respectively. RASi in combination with steroid (SUCRA 3.9\%) was the most effective in prevention of end-stage renal disease or doubling serum creatinine, followed by RASi monotherapy (SUCRA 38.4\%) and azathioprine combined with steroid (SUCRA 49.0\%). As for the occurrence of serious adverse events, azathioprine combined with RASi (SUCRA 88.0\%) and steroid plus RASi (SUCRA 74.6\%) showed the first and second highest incidence of adverse events, respectively. Conclusion: RASi combined with steroid demonstrated the most effective therapeutic approach for IgA nephropathy patients in terms of reducing proteinuria and stabilizing renal function.
\end{abstract}




\section{Kidney Blood Pressure Research}

Yang et al.: Agents to Improve IgA Nephropathy

\section{Introduction}

Immunoglobulin A(IgA) nephropathy was the most prevalentprimaryglomerulonephritis worldwide [1]. The deposition of IgA immune complexes in the mesangial area has been proposed as the critical factor in the onset of IgA nephropathy [2,3]. Approximately 15 to 20 percent of patients with IgA nephropathy will develop end-stage renal disease (ESRD) within 10 years, furthermore 30 to 40 percent within 20 years [4]. Therefore, effective treatment measures are needed for reducing proteinuria and to prevent the decline in kidney function. Various treatments have been studied in IgA nephropathy patients, such as renin-angiotensin system inhibitors (RASi), steroid, mycophenolate mofetil, azathioprine, cyclosporine A, and tonsillectomy, etc [1]. Unfortunately, the optimal treatment remains controversial.

The Kidney Disease Improving Global Outcomes (KDIGO) guidelines 2012 suggested the use of systemic glucocorticoids in IgA nephropathy patients with proteinuria level $>1 \mathrm{~g} / \mathrm{d}$ and estimated glomerular filtration rate (eGFR) $>50 \mathrm{ml} / \mathrm{min}$ per $1.73 \mathrm{~m}^{2}$ despite supportive care [5]. However, a recent retrospective study [6] for those with proteinuria more than $3.0 \mathrm{~g} / \mathrm{d}$ indicated that only $4 \%$ of individuals with RASi monotherapy reached a level $<1.0$ $\mathrm{g} / \mathrm{d}$ compared with $64 \%$ of those receiving steroid. More recently, the outcomes differed between The Therapeutic Evaluation of Steroids in IgA Nephropathy Global (TESTING) [7] and the Supportive versus Immunosuppressive Therapy for the Treatment of Progressive IgA Nephropathy (STOP-IgAN) [8] studies. Therefore, it is necessary to analyze the optimal therapeutic strategies for the IgA nephropathy patients with proteinuria more than $0.75 \mathrm{~g} / \mathrm{d}$.

To explore the efficacy and safety of different pharmacologic interventions in IgA nephropathy with proteinuria more than $0.75 \mathrm{~g} / \mathrm{d}$, we conducted the present network metaanalysis of randomized controlled trials (RCTs).

\section{Materials and Methods}

\section{Data Sources and Search Strategy}

We systematically searched the Cochrane Library, Embase, and PubMed database for articles up to August 1, 2018. No language restrictions were imposed during the searches. To identify studies involving relevant participants, we performed the search with keywords including the following terms: IgA nephropathy, immunoglobulin A nephropathy, and IgA nephritis. To identify studies involving therapies, we performed the search with keywords including the following terms: RASi, steroid, mycophenolate mofetil (MMF), azathioprine, cyclosporine A, and tonsillectomy. Electronic search strategies showed in the Supplementary Material S1 (for all supplementary material see www.karger.com/doi/10.1159/000496000). These 2 categories of keywords were combined using the Boolean operator "and". In this network meta-analysis, the presentations were based on the Preferred Reporting Items for reporting systematic reviews and metaanalyses (PRISMA) of studies that evaluate interventions [9].

\section{Selection Criteria}

We collected all RCTs on comparison of therapeutic effects of different drugs in IgA nephropathy patients with proteinuria more than $0.75 \mathrm{~g} / \mathrm{d}$. Inclusion criteria of studies: (a) study population was biopsyproven IgA nephropathy patients, (b) study design was RCTs, (c) the information of the interventions included placebo, RASi, steroid, MMF, tonsillectomy, azathioprine, and cyclosporine A, (d) the subjects with proteinuria or 24-hour urinary protein excretion more than $0.75 \mathrm{~g} / \mathrm{d}$ and eGFR $\geq 20 \mathrm{ml} / \mathrm{min}$ per $1.73 \mathrm{~m}^{2}$ or serum creatinine less than $3.5 \mathrm{mg} / \mathrm{dl}$, and (e) articles provided exact data of clinical remission, serious adverse events and/or ESRD or doubling serum creatinine level between patients with treatment group and control group.

In this network meta-analysis, clinical remission was defined as the achievement of proteinuria less than $1.0 \mathrm{~g} / \mathrm{d}$ or $50 \%$ greater of proteinuria reductions [5, 7]. The definition of ESRD was appeared one of these conditions: (a) serum creatinine level over $707 \mu \mathrm{mol} / \mathrm{L}$, and (b) need for maintenance dialysis or kidney transplantation [7]. Serious adverse events were defined according to the International Conference 


\section{Kidney Blood Pressure Research}

on Harmonization Guideline for Clinical Safety Data Management [7]. The definition of serious adverse events was appeared one of the following conditions: all-cause mortality, serious infections, liver function damage, gastrointestinal hemorrhage, new diabetes, osteonecrosis or fracture, and cardiovascular events.

Criteria for exclusion were as follows: (a) studies such as systemic reviews, case reports, comments, conference abstracts, editorials, etc, (b) the subjects with mild or severe proteinuria, or pathology confirmed as crescent, and (c) articles that had no definitions on clinical remission or renal function.

Included trials reported comparisons of 9 interventions (placebo, RASi, steroid, MMF, steroid plus RASi, tonsillectomy combined with steroid pulse therapy [TSP], azathioprine plus RASi, azathioprine steroid, and cyclosporine A in combination with steroid). The supportive and immunosuppressive interventions were made up according to the type of drugs, monotherapy or combination, regardless of dose.

\section{Data Extraction and Quality Assessment}

We assessed risk of bias in contributing studies with standard criteria. Two authors (Y-PP and W-Q) selected studies and extracted data independently. Disagreements were resolved through discussions or referral to the third author (X-GS). Our search strategy and selection criteria were described in the panel. Reference lists of identified RCTs were manually scanned to identify related research references at the same time as indicated in Fig. 1. The RCTs studies quality assessment were completed by using Review Manager 5.3 (Cochrane Collaboration, Oxford, UK) risk of bias tool including the following 7 domains: (a) random sequence generation (selection bias), (b) allocation concealment (selection bias), (c) performance bias, (d) detection bias, (e) attrition bias, (f) reporting bias, and (g) other bias.

\section{Data Synthesis and Analysis}

The network meta-analysis was performed using Stata (version 14.0, Stata SE), WinBUGS (version 1.4.3, MRC Biostatistics Unit, Cambridge, UK), and R software (version 3.5.1, R foundation for statistical computing). The odds ratio (OR) with corresponding 95\% confidence interval (CI) was utilized to compare different agents with respect to various clinical outcomes. We conducted conventional meta-analyses for treatments that were directly compared in RCTs by Bayesian random-effects model. Convergence was checked using the Brooks-Gelman-Rubin diagnostic and trace plots [10]. The stability of the results was obtained by sensitivity analyses with discarding each study sequentially. Ranking probabilities for all treatments were used the surface under the cumulative ranking area (SUCRA) [11]. Node-splitting approach was used to assess the inconsistency [12].

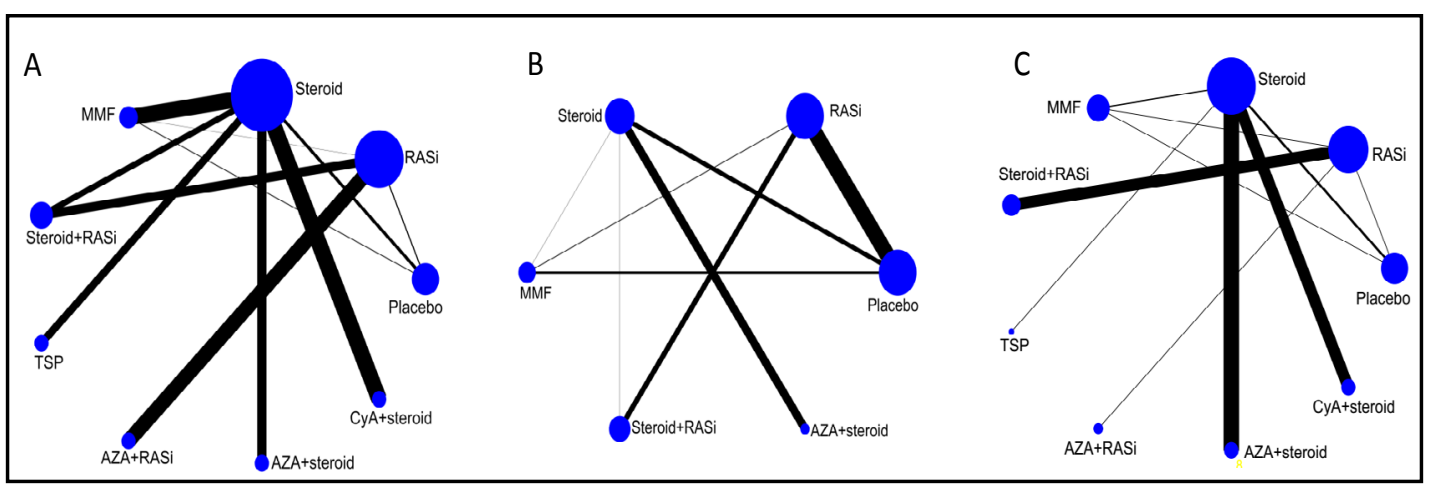

Fig. 1. Network plot of treatment comparisons for bayesian network meta-analysis. Lines represent trials comparing 2 classes of drug or drugs for (A) clinical remission, (B) ESRD and doubling of serum creatinine level, and (C) serious adverse events of IgA nephropathy. The size of the nodes (blue circles) corresponds to the sample size of interventions. Comparisons are linked with a line, of which the thickness corresponds to the number of trials that assessed the comparison. Abbreviations: MMF, mycophenolate mofetil; RAS, reninangiotensin system; TSP, tonsillectomy combined with steroid pulse therapy. 


\section{Kidney Blood Pressure Research}

\section{Results}

\section{Characteristics of Included Studies}

A total of 29 RCTs with 2517 participants were available for network meta-analysis, and the selection process details were showed in the Supplementary Material S2. Supplementary Material S3 summarized the essential baseline characteristic of these studies. The overall risk of bias was showed in Supplementary Material S4.

Network plot of treatment comparisons for bayesian network meta-analysis was showed in Fig. 1. The comparisons were connected by a straight line, of which the thickness corresponds to the number of trials that assessed the comparison. The size of the nodes (blue circles) corresponds to the sample size of interventions.

\section{Efficacy Outcomes-Clinical Remission}

Compared to placebo, the most effective treatments to induce remission were RASi monotherapy, steroid monotherapy, MMF monotherapy, RASi combined with steroid, TSP, azathioprine plus RASi, azathioprine in combination with steroid, and cyclosporine A plus steroid (OR of 6.1, 95\% CI 2.5 - 16.0; 4.2, 95\% CI 1.8 - 11.0; 5.6, 95\% CI 2.1 - 18.0; 19.0, 95\% CI 6.3 - 61.0; 20.0, 95\% CI 5.7 - 77.0; 46.0, 95\% CI 10.0 - 240.0; 5.7, 95\% CI 1.6 - 23.0; and $10.0,95 \%$ CI 3.0 - 38.0, respectively). RASi combined with steroid ranked the best treatment to induce clinical remission (Table 1).

RASi combined with steroid and TSP ranked the best and second (SUCRA of $82.9 \%$ and $80.5 \%$, respectively, Fig. 2A), followed by RASi combined with azathioprine, Cyclosporine A plus steroid and azathioprine combined with steroid (SUCRA of $67.6 \%, 66.2 \%$, and $44.2 \%$, respectively). Placebo was ranked the least effective treatment.

\section{Safety Outcomes}

ESRD or Doubling of Serum Creatinine. Compared to Placebo, RASi plus steroid and RASi displayed the lowest risks for ESRD or doubling of serum creatinine level (OR 0.04, 95\% CI $0.01-0.17$ and $0.01,95 \%$ CI $0.04-0.43$, respectively). There was no evidence that steroid monotherapy, MMF or azathioprine plus steroid showed different effects on ESRD or doubling serum creatinine compared to RASi or each other (Supplementary Material S5).

Table 1. Summary of results from network meta-analysis (on the lower triangle) and traditional pairwise meta-analysis (on the upper triangle) on clinical remission. On the lower triangle, the column-defining treatment is compared to the row-defining treatment, and odds ratios (OR) $<1$ favor the column-defining treatment. On the upper triangle, the row-defining treatment is compared to the column-defining treatment, and $\mathrm{OR}<1$ favor the row-defining treatment. To obtain ORs for comparisons in the opposite direction, reciprocals should be taken. Significant results are in bold. Direct comparisons within 2 inconsistent loops are underlined. Abbreviations: RASi, renin-angiotensin system inhibitors; MMF, mycophenolate mofetil; TSP, tonsillectomy combined with steroid pulse therapy; AZA, azathioprine; CyA, cyclosporine A

\begin{tabular}{|c|c|c|c|c|c|c|c|c|c|}
\hline $\begin{array}{c}\text { OR } \\
95 \% \mathrm{CI}\end{array}$ & Placebo & RASi & Steroid & MMF & $\begin{array}{l}\text { RASi + } \\
\text { steroid }\end{array}$ & TSP & $\mathrm{AZA}+\mathrm{RASi}$ & $\begin{array}{l}\text { AZA + } \\
\text { steroid }\end{array}$ & $\begin{array}{l}\text { CyA+ } \\
\text { steroid }\end{array}$ \\
\hline Placebo & & $\begin{array}{c}7.8 \\
(2.8,25.0)\end{array}$ & $\begin{array}{c}2.5 \\
(0.9,6.8)\end{array}$ & $\begin{array}{c}16 \\
(2.4,130.0)\end{array}$ & & & & & \\
\hline RASi & $\begin{array}{c}6.1 \\
(2.5,16.0)\end{array}$ & & & $\begin{array}{c}1.5 \\
(0.1,20.0)\end{array}$ & $\begin{array}{c}3.4 \\
(1.6,7.4)\end{array}$ & & & & \\
\hline Steroid & $\begin{array}{c}4.2 \\
(1.8,11.0)\end{array}$ & $\begin{array}{c}0.7 \\
(0.2,2.2)\end{array}$ & & $\begin{array}{c}0.9 \\
(0.3,2.6)\end{array}$ & $\begin{array}{c}2.0 \\
(0.2,26.0)\end{array}$ & & & & \\
\hline MMF & $\begin{array}{c}5.6 \\
(2.1,18.0)\end{array}$ & $\begin{array}{c}0.9 \\
(0.3,3.4)\end{array}$ & $\begin{array}{c}1.3 \\
(0.6,3.5)\end{array}$ & & & & & & \\
\hline $\begin{array}{l}\text { RASi + } \\
\text { steroid }\end{array}$ & $\begin{array}{c}19.0 \\
(6.3,61.0)\end{array}$ & $\begin{array}{c}3.2 \\
(1.5,6.5)\end{array}$ & $\begin{array}{c}4.6 \\
(1.3,16.0)\end{array}$ & $\begin{array}{c}3.5 \\
(0.8,13.0)\end{array}$ & & & & & \\
\hline TSP & $\begin{array}{c}20.0 \\
(5.7,77.0)\end{array}$ & $\begin{array}{c}3.3 \\
(0.8,15.0)\end{array}$ & $\begin{array}{c}4.7 \\
(1.9,12.0)\end{array}$ & $\begin{array}{c}3.5 \\
(0.9,13.0)\end{array}$ & $\begin{array}{c}1.0 \\
(0.2,5.2)\end{array}$ & & & & \\
\hline $\begin{array}{c}\mathrm{AZA}+ \\
\mathrm{RASi}\end{array}$ & $\begin{array}{c}46.0 \\
(10.0,240)\end{array}$ & $\begin{array}{c}7.4 \\
(2.2,27.0)\end{array}$ & $\begin{array}{c}11.0 \\
(2.0,61.0)\end{array}$ & $\begin{array}{c}8.2 \\
(1.3,47.0)\end{array}$ & $\begin{array}{c}2.4 \\
(0.6,11)\end{array}$ & $\begin{array}{c}2.3 \\
(0.3,16.0)\end{array}$ & & & \\
\hline $\begin{array}{c}\text { AZA + } \\
\text { steroid }\end{array}$ & $\begin{array}{c}5.7 \\
(1.6,23.0)\end{array}$ & $\begin{array}{c}0.9 \\
(0.2,4.3)\end{array}$ & $\begin{array}{c}1.4 \\
(0.5,3.6)\end{array}$ & $\begin{array}{c}1.0 \\
(0.3,3.7)\end{array}$ & $\begin{array}{c}0.3 \\
(0.1,1.5)\end{array}$ & $\begin{array}{c}0.3 \\
(0.1,1.1)\end{array}$ & $\begin{array}{c}0.1 \\
(0.01,0.9)\end{array}$ & & \\
\hline $\begin{array}{l}\text { CyA+ } \\
\text { steroid }\end{array}$ & $\begin{array}{c}10.0 \\
(3.0,38.0)\end{array}$ & $\begin{array}{c}1.7 \\
(0.4,7.2)\end{array}$ & $\begin{array}{c}2.4 \\
(1.0,5.9)\end{array}$ & $\begin{array}{c}1.8 \\
(0.5,6.3)\end{array}$ & $\begin{array}{c}0.5 \\
(0.1,2.5)\end{array}$ & $\begin{array}{c}0.5 \\
(0.1,1.8)\end{array}$ & $\begin{array}{c}1.0 \\
(0.07,1.1)\end{array}$ & $\begin{array}{c}1.8 \\
(0.5,6.7)\end{array}$ & \\
\hline
\end{tabular}




\section{Kidney Blood Pressure Research}

Fig. 2. Rankings of SUCRA for efficacy of treatments to induce end points in IgA nephropathy. (A) clinical remission, (B) ESRD and doubling of serum creatinine level, and (C) serious adverse events of IgA nephropathy. The graphs display the distribution of probabilities of treatment ranking from best through worst for each outcome. Ranking indicates the probability that drug class is first "best," second "best," etc. For example, RAS inhibitors plus antiplatelets and steroid combined with tonsillectomy were among the best treatments for inducing disease remission, while Placebo provided the lowest probability of disease remission (worst). On the other hand, the ranking suggests that MMF posed the highest risk for incurring ESRD or creatinine doubling (worst), while RAS inhibitors plus steroid incurred the lowest probability of ESRD or creatinine doubling (best). Abbreviations: MMF, mycophenolate mofetil; RAS, renin-angiotensin system, TSP, tonsillectomy combined with steroid pulse therapy.

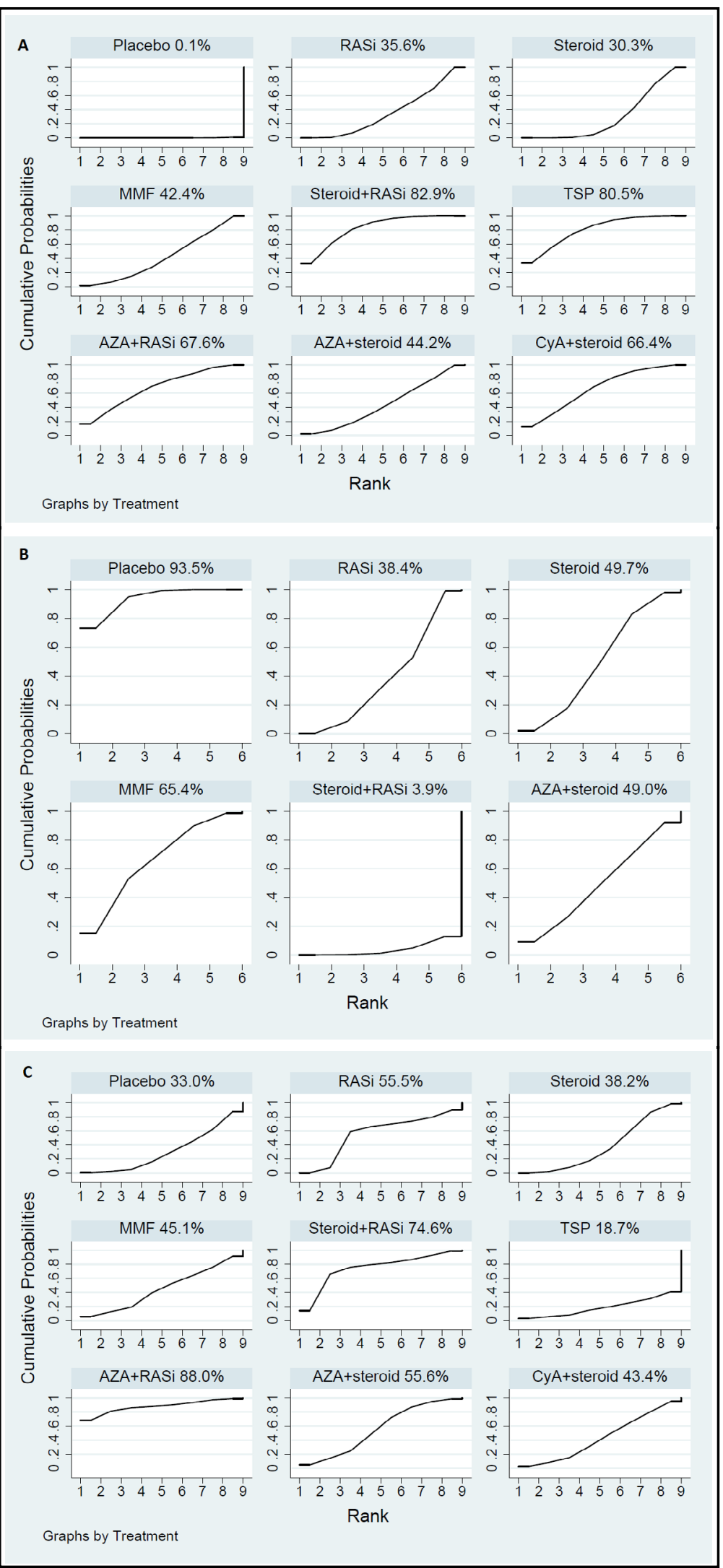




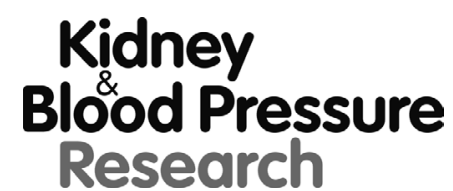
\begin{tabular}{l}
\hline Kidney Blood Press Res 2018;43:1890-1897 \\
\begin{tabular}{l|l}
\hline DOI: 10.1159/000496000 & (c) 2018 The Author(s). Published by S. Karger AG, Basel \\
Published onlIne: 14 December 2018 & ww.karger.com/kbr
\end{tabular} \\
\hline
\end{tabular}

Yang et al.: Agents to Improve IgA Nephropathy

As for the prevention of ESRD or doubling of serum creatinine level, RASi plus steroid (SUCRA 2.5\%) was the best treatment, followed by RASi monotherapy (SUCRA 37.4\%), steroid monotherapy (SUCRA 50.5\%). MMF was the least effective in preventing progression to ESRD (SUCRA 64.2\%) (Fig. 2B).

Serious adverse events. For serious adverse events, RASi combined with azathioprine (SUCRA 88.0\%) and RASi plus steroid (SUCRA 74.6\%) were ranked highly, followed by azathioprine plus steroid (SUCRA 55.6\%), RASi alone (SUCRA 55.5\%). In addition, there were non-significant differences among all interventions in the occurrence of serious adverse events, as illustrated in Fig. 2C and Supplementary Material S5.

\section{Sensitivity Analysis and Publication Bias}

We performed a subgroup analysis in IgA patients with proteinuria $>1 \mathrm{~g} / \mathrm{d}$, and there was non-significant difference compared with the group with proteinuria $>0.75 \mathrm{~g} / \mathrm{d}$. Firstly, 50,000 instances of iterations were increased to acquire satisfactory convergence, as showed in diagnostics and trace plots (Supplementary Material S6). Secondly, there was minimal heterogeneity in the results of clinical remission, ESRD, and serious adverse events, as showed in Supplementary Material S7. Then, Pair-wise and network meta-analysis estimates were similar in magnitude and testing without revealing evidence of inconsistency between direct and indirect treatment effects, as depicted in Supplementary Material S8. Publication bias was tested by funnel plot (Supplementary Material S9).

\section{Discussion}

The present network meta-analysis provided a unified hierarchy of evidence for all proteinuria lowering agents in IgA nephropathy patients with proteinuria more than 0.75 $\mathrm{g} / \mathrm{d}$. This network meta-analysis indicated that RASi plus steroid, steroid combined with tonsillectomy, azathioprine plus RASi and Cyclosporine A combined with steroid were superior to RASi monotherapy for inducing clinical remission. Compared to Placebo, RASi in combination with steroid and RASi monotherapy indicated lower risks for ESRD or doubling of serum creatinine level. Therefore, RASi plus steroid was in association with the lowest risks for worse renal function and was the best treatment to maintain disease remission. For serious adverse events, all interventions available were not significant in pair-wise and network meta-analysis.

KDIGO guideline recommend long-term RASi treatment with proteinuria more than 1 $\mathrm{g} / \mathrm{d}$ [5]. The current cumulative evidence showed that RASi had statistically significant effects in reducing proteinuria and protecting kidney function [13]. RASi was the fundamental for long-term treatment of progressive IgA nephropathy $[14,15]$. The KDIGO guidelines also suggest that IgA nephropathy patients with persistent proteinuria more than $1.0 \mathrm{~g} / \mathrm{d}$ despite 3 to 6 months of supportive care, and an eGFR $\geq 50 \mathrm{ml} / \mathrm{min}$ per $1.73 \mathrm{~m}^{2}$ accept glucocorticoids therapy [5]. The TESTING study [7] found that RASi combined with steroid was consistent with potential renal benefit but associated with an increased risk of serious adverse events. In the present study, however, the efficacy of RASi was less effective than RASi combined with steroid and TSP in inducing clinical remission.

The galactose-deficient IgA1 (GdIgA1) has a deficiency in the 0-glycan located at the hinge region of IgA1 $[3,16]$. GdIgA1 binds to its antibody to form a circulating immune complex that eventually deposits in the kidney and causes the renal function to deteriorate $[3,17]$. Nakata and his colleague found that serum GdIgA1 levels decreased more than half after tonsillectomy, which indicated that the palatine tonsils are probably major sites of GdIgA1-producing cells [18]. A meta-analysis suggested that TSP is helpful in inducing clinical remission and inhibiting the development of ESRD in IgA nephropathy patients [19].

Some multicenter RCTs showed that MMF did not decrease proteinuria significantly in patients with IgA nephropathy who had persistent proteinuria after intensive supportive 


\section{Kidney \\ Blood Pressure Research}

care [20, 21], but had fewer serious adverse events [21]. One meta-analysis by Xu et al. [22] suggested the evidence that did not support the use of MMF in moderately advanced IgA nephropathy. Floege and his colleague [23] offered a possible interpretation for the different outcomes observed in the Chinese subjects compared with Belgium and America. The present network meta-analysis indicated that MMF was associated with a higher risk to ESRD and less effective in clinical remission than RASi alone.

Stangou et al [24]. reported that treatment with steroid and azathioprine appeared to be an effective therapeutic approach for IgA nephropathy patients in terms of reducing proteinuria. Liu et al [25]. suggested that both steroids monotherapy and combined treatment with Cyclosporine A remarkably reduced the levels of proteinuria and ameliorated renal function in the IgA nephropathy patients. However, infection was the most serious complication during the treatment [25]. Our network meta-analysis showed that RASi combined with azathioprine, Cyclosporine A plus steroid and azathioprine combined with steroid also had a highly efficacy in clinical remission, but not in ameliorating renal function.

Our study has potential limitations. Firstly, we did not control for dosage in our analyses. And the usage of steroid was not uniform, some with direct oral prednisone, and the others methylprednisolone pulse therapy. Secondly, the effect of pathological types on treatment was not considered. Thirdly, although the heterogeneity in the network analyses was low, it is likely that the low power to detect heterogeneity is due to limited data. Hence, there is needed to perform several larger, multicenter RCTs to acquire more robust results.

\section{Conclusion}

In conclusion, the current network meta-analysis suggested that RASi combined with steroid was the best therapeutic option in terms of reducing proteinuria and stabilizing renal function in IgA nephropathy patients with proteinuria $>0.75 \mathrm{~g} / \mathrm{d}$. Nonetheless, RASi plus steroid was associated with the highly risk of serious adverse events.

\section{Acknowledgements}

This work was supported by the National Natural Science Foundation of China (No. H0517/81560132), the Supporting Project for the Foregoers of Main Disciplines of Jiangxi Province (No. 20162BCB22023), and the "5511" Innovative Drivers for Talent Teams of Jiangxi Province (No. 20165BCB18018).

\section{Disclosure Statement}

The authors declare that they have no competing interests.

\section{References}

$>1$ Wyatt RJ, Julian BA: IgA nephropathy. N Engl J Med 2013;368:2402-2414.

2 Yeo SC, Cheung CK, Barratt J: New insights into the pathogenesis of IgA nephropathy. Pediatr Nephrol 2018;33:763-777.

3 Zhao YF, Zhu L, Liu LJ, Shi SF, Lv JC, Zhang H: Pathogenic role of glycan-specific IgG antibodies in IgA nephropathy. BMC Nephrol 2017;18:301.

4 Magistroni R, D’Agati VD, Appel GB, Kiryluk K: New developments in the genetics, pathogenesis, and therapy of IgA nephropathy. Kidney Int 2015;88:974-989. 


\section{Kidney \\ Blood Pressure Research}

5 Radhakrishnan J, Cattran DC: The KDIGO practice guideline on glomerulonephritis: reading between the (guide) lines-Application to the individual patient. Kidney Int 2012;82:840-856.

-6 Tesar V, Troyanov S, Bellur S, Verhave JC, Cook HT, Feehally J, Roberts IS, Cattran D, Coppo R: Corticosteroids in IgA Nephropathy: A Retrospective Analysis from the VALIGA Study. J Am Soc Nephrol 2015;26:2248-2258.

-7 Lv J, Zhang H, Wong MG, Jardine MJ, Hladunewich M, Jha V, Monaghan H, Zhao M, Barbour S, Reich H, Cattran D, Glassock R, Levin A, Wheeler D, Woodward M, Billot L, Chan TM, Liu ZH, Johnson DW, Cass A, et al. Effect of Oral Methylprednisolone on Clinical Outcomes in Patients With IgA Nephropathy: The TESTING Randomized Clinical Trial. JAMA 2017;318:432-442.

-8 Rauen T, Eitner F, Fitzner C, Sommerer C, Zeier M, Otte B, Panzer U, Peters H, Benck U, Mertens PR, Kuhlmann U, Witzke O, Gross O, Vielhauer V, Mann JF, Hilgers RD, Floege J: Intensive Supportive Care plus Immunosuppression in IgA Nephropathy. N Engl J Med 2015;373:2225-2236.

-9 Liberati A, Altman DG, Tetzlaff J, Mulrow C, Gøtzsche PC, Ioannidis JP, Clarke M, Devereaux PJ, Kleijnen J, Moher D: The PRISMA statement for reporting systematic reviews and meta-analyses of studies that evaluate health care interventions: explanation and elaboration. Ann Intern Med 2009;151:W65-94.

$\checkmark 10$ Brooks SP, Gelman A: General methods for monitoring convergence of iterative simulations. J Comput Graph Stat 1998;7:434-455.

11 Salanti G, Ades AE, Ioannidis JP: Graphical methods and numerical summaries for presenting results from multipletreatment meta-analysis: an overview and tutorial. J Clin Epidemiol 2011;64:163-171.

$>12$ Spiegelhalter DJ, Best NG, Carlin BP, Angelika VDL: Bayesian measures of model complexity and fit. Journal of the Royal Statistical Society 2002;64:583-639.

13 Cheng J, Zhang W, Zhang XH, He Q Tao XJ, Chen JH: ACEI/ARB therapy for IgA nephropathy: a meta analysis of randomised controlled trials. Int J Clin Pract 2009;63:880-888.

14 Woo KT, Lau YK, Zhao Y, Liu FE, Tan HB, Tan EK, Stephanie FC, Chan CM, Wong KS: Disease progression, response to ACEI/ATRA therapy and influence of ACE gene in IgA nephritis. Cell Mol Immunol 2007;4:227232.

15 Moriyama T, Iwasaki C, Tanaka K, Ochi A, Shimizu A, Shiohira S, Itabashi M, Takei T, Uchida K, Tsuchiya K, Nitta K: Effects of combination therapy with renin-angiotensin system inhibitors and eicosapentaenoic acid on IgA nephropathy. Intern Med 2013;52:193-199.

-16 Reily C, Ueda H, Huang ZQ, Mestecky J, Julian BA, Willey CD, Novak J: Cellular signaling and production of galactose-deficient IgA1 in IgA nephropathy, an autoimmune disease. J Immunol Res 2014;2014:197548.

17 Novak J, Julian BA, Tomana M, Mestecky J: IgA glycosylation and IgA immune complexes in the pathogenesis of IgA nephropathy. Semin Nephrol 2008;28:78-87.

18 Nakata J, Suzuki Y, Suzuki H, Sato D, Kano T, Yanagawa H, Matsuzaki K, Horikoshi S, Novak J, Tomino Y: Changes in nephritogenic serum galactose-deficient IgA1 in IgA nephropathy following tonsillectomy and steroid therapy. PLoS One 2014;9:e89707.

19 Duan J, Liu D, Duan G, Liu Z: Long-term efficacy of tonsillectomy as a treatment in patients with IgA nephropathy: a meta-analysis. Int Urol Nephrol 2017;49:103-112.

-20 Tang SC, Tang AW, Wong SS, Leung JC, Ho YW, Lai KN: Long-term study of mycophenolate mofetil treatment in IgA nephropathy. Kidney Int 2010;77:543-549.

-21 Hou JH, Le WB, Chen N, Wang WM, Liu ZS, Liu D, Chen JH, Tian J, Fu P, Hu ZX, Zeng CH, Liang SS, Zhou ML, Zhang HT, Liu ZH: Mycophenolate Mofetil Combined With Prednisone Versus Full-Dose Prednisone in IgA Nephropathy With Active Proliferative Lesions: A Randomized Controlled Trial. Am J Kidney Dis 2017;69:788-795.

22 Xu G, Tu W, Jiang D, Xu C: Mycophenolate mofetil treatment for IgA nephropathy: a meta-analysis. Am J Nephrol 2009;29:362-367.

23 Floege J, Eitner F: Current therapy for IgA nephropathy. J Am Soc Nephrol 2011;22:1785-1794.

24 Stangou M, Ekonomidou D, Giamalis P, Liakou H, Tsiantoulas A, Pantzaki A, Papagianni A, Efstratiadis G, Alexopoulos E, Memmos D: Steroids and azathioprine in the treatment of IgA nephropathy. Clin Exp Nephrol 2011;15:373-380.

25 Liu H, Xu XL, Fang Y, Ji J, Zhang XY, Yuan M, Liu CF, Ding XQ: Comparison of Glucocorticoids Alone and Combined with Cyclosporine A in Patients with IgA Nephropathy: A Prospective Randomized Controlled Trial. Intern Med 2014;53:675-681. 